\title{
Multi-function microfluidic platform for sensor integration
}

\author{
Fernandes, Ana C.; Semenova, Daria; Panjan, Peter; Sesay, Adama M.; Gernaey, Krist V.; Krühne, Ulrich
}

Published in:

New Biotechnology

Link to article, DOI:

10.1016/j.nbt.2018.03.001

Publication date:

2018

Document Version

Peer reviewed version

Link back to DTU Orbit

Citation (APA):

Fernandes, A. C., Semenova, D., Panjan, P., Sesay, A. M., Gernaey, K. V., \& Krühne, U. (2018). Multi-function microfluidic platform for sensor integration. New Biotechnology, 47, 8-17.

https://doi.org/10.1016/j.nbt.2018.03.001

\section{General rights}

Copyright and moral rights for the publications made accessible in the public portal are retained by the authors and/or other copyright owners and it is a condition of accessing publications that users recognise and abide by the legal requirements associated with these rights.

- Users may download and print one copy of any publication from the public portal for the purpose of private study or research.

- You may not further distribute the material or use it for any profit-making activity or commercial gain

- You may freely distribute the URL identifying the publication in the public portal

If you believe that this document breaches copyright please contact us providing details, and we will remove access to the work immediately and investigate your claim 


\title{
Multi-function microfluidic platform for sensor integration
}

\author{
Ana C. Fernandes ${ }^{1 *}$, Daria Semenova ${ }^{1}$, Peter Panjan ${ }^{2}$, Adama M. Sesay ${ }^{2}$, Krist V. Gernaey ${ }^{1}$ and \\ Ulrich Krühne ${ }^{1}$
}

${ }^{1}$ Process and Systems Engineering Center (PROSYS), Department of Chemical and Biochemical Engineering, Technical University of Denmark, Building 229, 2800 Kgs. Lyngby, Denmark

${ }^{2}$ Measurement Technology Unit (MITY), Teknologiapuisto PL 127, Kehräämöntie 7, 87400 Kajaani, Finland

*corresponding author e-mail: ancafe@kt.dtu.dk

\begin{abstract}
The limited availability of metabolite-specific sensors for continuous sampling and monitoring is one of the main bottlenecks contributing to failures in bioprocess development.

Furthermore, only a limited number of approaches exist to connect currently available measurement systems with high throughput reactor units. This is especially relevant in the biocatalyst screening and characterization stage of process development.

In this work, a strategy for sensor integration in microfluidic platforms is demonstrated, to address the need for rapid, cost-effective and high-throughput screening in bioprocesses. This platform is compatible with different sensor formats by enabling their replacement and was built in order to be highly flexible and thus suitable for a wide range of applications. Moreover, this re-usable platform can easily be connected to analytical equipment, such as HPLC, laboratory-scale reactors or other microfluidic chips through the use of standardized fittings. In addition, the developed platform includes a two-sensor system interspersed with a mixing channel, which allows the detection of samples that might be outside the first sensor's range of detection, through dilution of the sample solution up to 10 times.

In order to highlight the features of the proposed platform, inline monitoring of glucose levels is presented and discussed. Glucose was chosen due to its importance in biotechnology as a relevant substrate. The platform demonstrated continuous measurement of substrate solutions for up to $12 \mathrm{~h}$. Furthermore, the influence of the fluid velocity on substrate diffusion was observed, indicating the need for in-flow calibration to achieve a good quantitative output.
\end{abstract}

Keywords: microfluidics, sensor integration, screening platform, in-flow glucose monitoring, sample dilution

\section{Introduction}

Enzyme and strain screening, along with the optimization of bioprocess operation conditions are key steps in the development of a biotechnological process [1]. The large number and variety of enzyme/strain candidates and corresponding optimal conditions result in an expensive and time-consuming development process. Currently, high throughput methods [2],[3], mainly performed on microtiter plates and shake flasks, are the preferred experimental 
test platforms. These allow for high throughput due to the use of easily programmed and flexible automatic fluid handling systems [4], but offer a reduced amount of analytical data, leading to an overall limited knowledge on the process and enzyme/strains [5].

Microfluidic systems show a considerable potential in screening applications and online monitoring. The variety of achievable geometries, high surface to volume ratio and diversity of sensing technologies available at small scale, make them highly attractive tools for bioprocess development. Their attractiveness is further increased due to the possibility of parallelization, enabling an increase in throughput while occupying a smaller area. Moreover, a higher control of operation conditions, especially flow, permits a good definition of gradients of the components involved, which can be further improved with automation. However, due to the challenges involved in sensor development and integration, microfluidic systems are still application-specific, since they are typically too rigid to address the needs of different bioprocesses [6]. Therefore, a change in the target sample (e.g. type of sample)or the sensor technology or flow conditions used, or the need for integration with other systems, require a redesign and the fabrication of a new platform. This greatly reduces the microfluidics range of applications, thus standing in the way of the widespread use of microfluidic systems.

One of the main contributors to this microfluidic specificity is sensor integration. The sensors used in each platform need to be highly specific for a target compound, or a range of target compounds, and are optimized for a given set of temperature, sample or flow conditions, consequently being often limited in application. Thus, the applied strategy for sensor integration in a microfluidic platform depends on the detected sample, the type of sensor and the materials that form the sensor and the platform. Sensor integration can be performed by either fabrication of the sensor along with the microfluidic structure or by enclosing the sensor within the microfluidics following fabrication. While the former usually involves a permanent integration of the sensor(s), the latter can be designed towards an exchangeable and more flexible sensor format.

The first approach is generally applied for silicon and glass substrates using thin film technologies [7], [8], [9]. The sensors are deposited onto the channel surface using technologies similar or compatible with those used for the microstructure fabrication, such that the fabrication of both structures can be performed simultaneously or sequentially. In polymeric substrates these techniques can also be used, but differences between the thermal expansion coefficients of the metals and the polymer where deposition occurs may lead to metal layer stress and sensor cracking. Another sensor layer deposition method is the thick-film method, in which a paste containing metal particles or conducting ink (e.g. carbon or graphite ink) is spread on a screen with the geometrical pattern of the contact (e.g. electrode) or sensor (e.g. optical). This technique can also be used to print sensors onto paper strips, forming screenprinted electrodes, and to integrate biomolecules in the electrodes paste, directly printing biomolecules for identification of specific analytes [10], [11]. Sensors fabricated with other technologies (spray-coating [12], coating coupled with embossing [13], photolithography and wet etching [14]) can also be irreversibly encapsulated into a microfluidic channel. Irreversible encapsulation guarantees a leakage and contamination-free operation of the device (after sterilization or when assembled in a clean-room environment), and it is the most frequently 
used approach. However, a platform design that enables the substitution of the sensors in use and the integration of different types of sensors would have a wider applicability. This can be achieved using platforms where the channel is not irreversibly bonded and can then be easily and frequently opened [15], [16], [17].

Reversible encasing of the sensors can be performed through the use of a chip holder (e.g. with mechanical clamping [18], [17]) which applies enough pressure for allowing leakage-free flows. A reversible encapsulation can facilitate sensor or channel surface functionalization and cleaning due to the easy access to these surfaces. If the sensor is not irreversibly bonded or encased in the device, it can be further re-used in other microfluidic platforms. The cost of such devices can be consequently reduced, since the sensors are often the most expensive component of a microfluidic platform. Another strategy allows for replacing the used sensors inbetween experiments. In this case the sensors are inserted inside the channel of the device through cavities [19], [20], which enables a re-use of the microfluidic system for different target molecules by allowing the introduction of different sensors. Nevertheless, in both approaches, one of the significant challenges is the integration between the sensor and the channel, which must be performed in order to avoid leakage at the interface [10]. Furthermore, when designing a microfluidic system, it is important to consider how the connection to external devices will be performed, both in terms of fluid handling and data or signal acquisition. Standardizing both the microfluidic design and the connectors enables an easy integration with other chips or equipment, as well as chip modification for different applications [21]. The connection to external devices also depends on the type of sample used $[22]$.

In this study, we focus on addressing the current need for adaptable screening platforms with integrated sensors. We propose a flexible multi-function platform, with disposable and changeable sensors, being easily integrated with other microfluidic platforms through a "plugand-play" approach by using standardized finger-tight fittings. The platform can be easily connected to external equipment (e.g. syringe pumps, HPLC), while offering the advantage of in-line use, thus not interfering with the reaction vessel. The choice of material, the thermopolymeric Poly(methyl methacrylate) (or PMMA), and fabrication technology (laser ablation) considered both design flexibility and costs. PMMA and laser ablation allow a fast optimization cycle from design, to fabrication and testing with fluids. Furthermore, since PMMA is a widely available biocompatible and cheap material, it enables a low cost device fabrication with good reproducibility.

The classical example of the catalysed oxidation reaction of glucose by glucose oxidase is chosen as the model reaction to highlight the monitoring capabilities of the developed microfluidic platform. In order to track the changes in analyte concentration inline, first generation amperometric glucose biosensors were chosen for further integration into the developed microfluidic device. Amperometric electrodes were employed due to their wide applicability [23], [24], [25], [26,27], compactness, stability and low price. 


\section{Materials and Methods}

\section{Reagents and materials}

Glucose oxidase (EC 1.1.3.4, type VII, from Aspergillus niger, $\geq 100,000 \mathrm{U} / \mathrm{g}$ solid) and bovine serum albumin (BSA) were obtained from Sigma (St. Louis, MO, USA). Nafion ${ }^{\circledR} 117$ solution $(\sim 5 \%(\mathrm{v} / \mathrm{v})$ in a mixture of lower aliphatic alcohols and water) and iron (III) chloride (anhydrous, 99.99\%) were obtained from Aldrich (Steinheim, Germany). Potassium hexacyanoferrate (III) (ACS reagent, $\geq 99.0 \%$ ), glutaraldehyde solution (25\% (v/v)), Phosphate buffer saline (PBS) were obtained from Sigma - Aldrich. Yeast extract and peptone were bought from BD (France) and dextrose from Difco (MI, USA). The hydrochloric acid to set the $\mathrm{pH}$ of the fermentation medium was diluted to $10 \mathrm{mM}$ from concentrate (Merck, Germany). DGlucose (anhydrous) was provided by Fluka (Loughborough, UK). Mono - and di-potassium hydrogen phosphate (anhydrous) were obtained from Merck (Darmstadt, Germany).

All the solutions for sensor preparation were prepared with $0.1 \mathrm{M}$ phosphate buffer $+0.1 \mathrm{M}$ $\mathrm{KCl}, \mathrm{pH}$ 6. Standard solutions were prepared daily in the same buffer. All other solutions were prepared in $0.1 \mathrm{M}$ phosphate.

Customized screen-printed electrodes (SPEs) on polymeric substrate (DRP-PW-110DGPHOX) were produced by DropSens (Llanera, Spain), while the SPEs on polyester substrate (ItalSens IS-C) were acquired from PalmSens (Utrecht, The Netherlands). The DRP-PW-110DGPHOX SPEs are made of a thicker polymer than than the IS-C SPES. Each sensor is composed of three screen-printed electrodes: (i) the carbon working electrode (with graphene oxide layer in the case of the DRP-PW-110DGPHOX electrodes); (ii) the carbon counter electrode; and, (iii) the silver reference electrode. The diameter of the working electrode in the DRP-PW110DGPHOX SPE is $0.4 \mathrm{~cm}$, which results in an apparent geometric area of $0.126 \mathrm{~cm}^{2}$, while in the IS-C SPE the diameter is $0.3 \mathrm{~cm}$ with an apparent geometric area of $0.07 \mathrm{~cm}^{2}$. The extrusion PMMA sheets with thickness of $2 \mathrm{~mm}$ were acquired from Nordisk Plast (Assentoft, Denmark). The PMMA plates were patterned using laser ablation with a CMA4030 Laser Engraving machine from GD Han's Yueming Laser Technology co., Ltd (Guangdong, China). Chemical bonding of the PMMA plates was achieved using an anhydrous chloroform solution, $\geq 99 \%, 288306$ from Sigma-Aldrich. Adhesive assembly and integration of the sensors on the platform were performed with a $140 \mu \mathrm{m}$ thick mcs-foil 008 from microfluidic ChipShop (Jena, Germany). The PDMS thin sheets used as gaskets were fabricated using the elastomer and curing agent kit of Sylgard ${ }^{\circledR} 184$ from Dow Corning (Auburn, Michigan). The two $10 \mathrm{~mm}$ thick acryl plates that form the custom-made holder were from Rias A/S (Roskilde, Denmark). The holder was completed with two outer $5 \mathrm{~mm}$ thick SS304 stainless steel plates from Sanistål (Aalborg, Denmark). Flangeless polypropylene (PP) fingertight 1.5875 mm (ID) fittings (XP-201) and flangeless ferrules (P200X) from Upchurch Scientific ${ }^{\circledR}$ (Washington, USA) were used to connect polytetrafluoroethylene (PTFE) $1.5875 \mathrm{~mm}$ (OD) x $1 \mathrm{~mm}$ (ID) tubing (S 1810-12) from Bohlender (Grünsfeld, Germany).

\section{Microfluidic Platform Fabrication and Sensor Integration}


A microfluidic system containing two inlets, two sensing/ measurement chambers, for sensor integration, and one mixing unit was developed and is presented in Figure 1. Figure 1(a) shows a schematic of the microfluidic platform. As illustrated, one of the inlets of the system is connected to the first chamber, where the sensor used as reference is located. The second inlet is connected to the mixing chamber, which serves here as a dilution unit but can also be used as a reactor or target labelling unit. The second chamber is positioned after the mixing unit. The sensor integrated in this chamber is the one used for sample quantification and is thus designated detection sensor. Figure 1 (b) and (c) display one more feature of this system. It involves the use of generic rectangular pockets at the bottom plate of the device with direct access to the sensing chambers. The sensor can thus be directly placed in the chamber and covered with a thin polymeric gasket. The system is then closed by placing PMMA inserts in the pockets and sealing the whole platform with help of an outside mechanical holder system.

The design of the device was performed in SolidWorks 2015 (Dassault Systèmes SolidWorks Corporation, Waltham, Massachusetts, USA). This microfluidic platform consists of four PMMA plates engraved by laser ablation. After engraving, the plates were thoroughly washed with deionised water, dried and then placed in an oven at $80^{\circ} \mathrm{C}$ for 1 hour to release thermal stress before proceeding with assembly. The PMMA plates were assembled by adhesion with a double-sided pressure sensitive adhesive tape (DAT) mcs-foil 008 from microfluidic ChipShop (Jena, Germany). The sensors were fixed in place using thin PDMS gasket sheets, sealing the sensor around the sensing chamber. The cooled plates were glued together and then pressurized with clamps and placed in an oven at $80{ }^{\circ} \mathrm{C}$ for at least $1 \mathrm{~h}$ to remove possible air bubbles disturbing the adhesion. The PDMS gaskets were fabricated by mixing in a SpeedMixer ${ }^{\mathrm{TM}}$ DAC 150.1 FVZ-K from Synergy Devices Limited (High Wycombe, UK). A 1:10

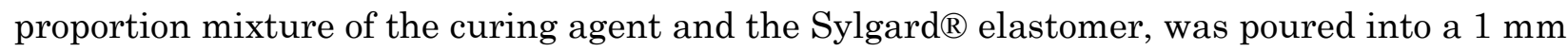
PMMA mold with the shape of the sensor pockets and curing it in the oven for $1 \mathrm{~h}$ at $70{ }^{\circ} \mathrm{C}$. Once the chip was assembled, the sensors were placed on the sensor pockets, the PDMS gaskets were placed on top and followed by a piece of PMMA. The microfluidic platform with integrated sensors was then positioned in an 'in-house' fabricated casing, which provided the necessary pressure to achieve leakage-free flow. The holder was built of two acrylic plates of 100x100 $\mathrm{mm}^{2}$ that were micromilled in order to have a cavity with the chip's size and two outer SS304 stainless steel plates with a thickness of $5 \mathrm{~mm}$, to avoid bending of the acryl plates when screwing the assembled holder together. This holder provides enough uniform pressure to avoid leakage of the microfluidic platform.

\section{Mixing unit CFD optimization and characterization}

Several passive mixing geometries were designed in SolidWorks 2015. The drawings of these geometries are presented in Figure $2 \mathrm{~S}$ in the supplementary information section. Each geometry's mixing characteristics were studied with the help of a computational fluid dynamic simulation software, ANSYS-CFX Version 16.2 (Canonsburg, Pennsylvania, USA). For this purpose, the three-dimensional designs were imported into a meshing software, ANSYS ICEM CFD ${ }^{\circledR} 16.0$ (Canonsburg, Pennsylvania, USA), where discretization of the geometries was performed. Discretization divides the geometry into smaller elements, in which the required momentum, energy and mass balance equations were solved by ANSYS-CFX. The number of 
elements of the 5 simulated structures varied between 845 and 1185 elements/ $\mu \mathrm{L}$, except for design (e) which had 9925 elements/ $\mu \mathrm{L}$. A structured mesh provides a more reliable and accurate output with respect to the results and can reduce the simulation time. However, it is more complicated to define and requires longer preparation time. Thus, as a first approach, all geometries were simulated with unstructured mesh elements using tetrahedral features for the mesh. The best designs ((a) and (e)) were then simulated with a structured hexahedron mesh. All geometries were simulated at the target flow conditions (steady state with laminar flow, outlet as an opening and no slip wall conditions) in the integrated microfluidic platform considering a fluid with the same properties as water $\left(\right.$ at $25{ }^{\circ} \mathrm{C}$ ) where a liquid tracer with diffusivity of $1 \times 10^{-10} \mathrm{~m}^{2} \mathrm{~s}^{-1}$ was introduced. The average inlet velocity, at the reference sensor, was $1 \times 10^{-3} \mathrm{~m} \mathrm{~s}^{-1}$, while the velocity of the other inlet was varied depending on the simulated degree of dilution. The geometries were evaluated by the average tracer amount at the outlet, as well as the gradient of its concentration. The best performing geometries were then built using the same procedure as described above for the microfluidic platform. Subsequently they were qualitatively tested using food colouring dyes at the simulated flow-rates. The geometry that achieved the desired 1:9 dilution with no gradient of tracer at the outlet (design (e)) was then used in the final microfluidic platform design.

\section{Sensor}

The biosensors used consist of an SPE with immobilized layers of glucose oxidase (GOx), as biological recognition element, and the redox mediator Prussian Blue (PB) on top of the working electrode. When the biosensor is in direct contact with the analyte solution, the substrate (glucose) diffuses through a polymeric matrix and binds to the enzymatic layer. The biocatalytic transformation involves the reduction of the flavin groups (FAD) of the enzyme (glucose oxidase) with further production of the reduced form (FADH2), followed by reoxidation by molecular oxygen $[28,29]$. The hydrogen peroxide, produced during the abovementioned conversion step, acts in the selective electrocatalytic reaction with Prussian Blue $[30,31]$. The amperometric detection of these substances is performed at the carbon working electrode (with and without graphene oxide), assisted by the counter electrode and the silver reference electrode. The measured current can therefore be directly related to the concentration of glucose in the bulk solution and used for quantification.

\section{Sensor Preparation}

The Prussian Blue (PB) modification of SPEs and deposition procedures were adopted from the procedures developed by Ricci et al. [32]. Freshly prepared precursor solutions of $0.1 \mathrm{M}$ potassium ferricyanide $\left(\mathrm{K}_{3} \mathrm{Fe}(\mathrm{CN})_{6}\right)$ in $10 \mathrm{mM} \mathrm{HCl}$ and $0.1 \mathrm{M}$ ferric chloride in $10 \mathrm{mM} \mathrm{HCl}$ were mixed and applied ( $10 \mu \mathrm{l}$ droplet) in the working electrode area in a 1:1 proportion in the DRP-PW-110DGPHOX SPEs. In the IS-C SPEs a 2:1 proportion was used for the detection sensor and a 1:2 proportion for the reference sensor.

Glucose oxidase (GOx) immobilization was performed in a Nafion matrix via a cross-linking method previously described by Ricci et al. [32]. A drop of $2.5 \mu \mathrm{l}$ of glutaraldehyde ( $1 \%$ (v/v) diluted in water) was placed on top of the working electrode area of the PB-modified electrodes and left to dry for $30 \mathrm{~min}$ at room temperature. A drop of $3 \mu \mathrm{l}$ of the enzymatic-Nafion 
membrane was then applied on the modified working electrode and placed to dry overnight in a climate chamber at $8^{\circ} \mathrm{C}$ with $40 \%$ of humidity. The enzymatic mixture had the following composition per sensor: GOx (0.074 U in phosphate buffer), BSA (5 vol. \% diluted in water) and Nafion ( 5 vol. \% diluted in water). All the components of the enzymatic layer were mixed in a 1:1:1 proportion. The complete glucose biosensors were stored dry at $4^{\circ} \mathrm{C}$ until use.

\section{Sensor Characterization}

Cyclic voltammetry (CV) and amperometric measurements (AM) were carried out using a MultiEmStat with a DRP-CAST1X8 interface (DropSens, Llanera, Spain) for 8 independent electrodes under MultiTrace Software 3.4 control (PalmSens, Utrecht, The Netherlands). Fluid flow was maintained by Cavro® XL 3000 syringe pumps from Tecan (Männedorf, Switzerland). The behaviour of the PB layer was studied by cycling in a potential range between -0.5 and $+1.2 \mathrm{~V}$ with a scan rate of $50 \mathrm{mV} / \mathrm{s}$ (presented in Figure $1 \mathrm{~S}$ in the supplementary information section). CV measurements were performed in droplets using phosphate buffer solution (0.1 M phosphate + 0.1 M KCl, pH 6).

The chronoamperometric measurements were performed in a droplet (50 $\mu$ l of the glucose solution) at an applied potential of $-0.16 \mathrm{~V}$ (graphene oxide-based sensors) or $-0.04 \mathrm{~V}$ (graphite-based sensors) versus a screen printed internal silver reference electrode. In flow measurements were carried out at the flowrates used for the mixing/dilution chamber characterization. The flow rates during characterization varied between 0.25 and $10 \mu \mathrm{L} / \mathrm{s}$. When a stable baseline current was reached (30 s and $60 \mathrm{~s}$, for graphene oxide and graphite sensors respectively) with phosphate buffer solution ( $0.1 \mathrm{M}$ phosphate $+0.1 \mathrm{M} \mathrm{KCl}, \mathrm{pH} 6)$, different analyte concentrations were tested and the measurements were recorded. In the droplet characterisation of the glucose biosensors, the drop of $50 \mu \mathrm{l}$ of known glucose concentration $(0.1 \mathrm{mM}-8 \mathrm{mM})$ was placed on top of all the electrodes and the current was recorded until it reached a stable value. When the sensors were characterized in flow, a volume corresponding to 3 times the internal volume of the microfluidic platform was first pumped to guarantee that only the target concentration was inside the system. Data was retrieved after stabilization of the signal.

\section{Continuous monitoring of diluted glucose solutions}

To perform the dilution inside the microfluidic platform, a $3 \mathrm{mM}$ glucose solution was introduced through the inlet connected to the first sensing chamber (working in this case as a reference sensor - see Figure 1 (a)). A buffer solution (the same as that used during measurements of the zero point during sensor characterization) was introduced in the second inlet (connected to the mixing/dilution chamber). The flowrate for substrate introduction was kept the same $(1 \mu \mathrm{L} / \mathrm{s})$ while the flow rate of the buffer solution was increased in order to achieve the correct dilution as presented in Table 1. The diluted solution was collected at the outlet of the microfluidic platform and measured in an HPLC for validation of the mixing unit and compared with the data retrieved from the integrated sensors.

\section{Evaluation of sensor performance with fermentation samples}


To evaluate the performance of the integrated electrochemical sensors for monitoring glucose concentration in fermentation samples, graphite sensors similar to those integrated in the microfluidic platform were used at-line to follow the consumption of glucose during the growth phase of a yeast fermentation culture. A 3D printed, magnetically stirred microbioreactor with an internal volume of $1 \mathrm{~mL}$ was used for the proof-of-concept glucose measurements of glucose consumption in fermentation. Yeast peptone dextrose (YPD) medium was prepared by dissolving $1 \mathrm{~g}$ yeast extract, $2 \mathrm{~g}$ dextrose and $2 \mathrm{~g}$ of peptone in $100 \mathrm{~mL}$ of deionized water, followed by stirring and heating until everything was dissolved. The $\mathrm{pH}$ value was adjusted to 4.5 by the addition of $10 \mathrm{mM} \mathrm{HCl}$. The medium was placed into the above-mentioned microbioreactor and heated at $90^{\circ} \mathrm{C}$ overnight for sterilization. $10 \mathrm{mg}$ of dry baking yeast (Saccharomyces cerevisiae acquired at the local grocery store) was added into the microbioreactor after sterilization. At this point, a $20 \mu \mathrm{L}$ sample was taken in a sterile manner for the determination of a baseline with the glucose biosensor before spiking the fermentation broth with highly concentrated glucose (aqueous) solution to elevate the glucose levels within the microbioreactor to $20 \mathrm{~g} / \mathrm{L}$. Another sample $(20 \mu \mathrm{L})$ was taken at this point to determine the sensitivity of the glucose biosensor, followed by continuous sampling every $30 \mathrm{~min}$ for the glucose measurements during the fermentation in an incubator set at $30^{\circ} \mathrm{C}$. To ensure 1:50 dilution, $980 \mu \mathrm{L}$ of PBS buffer was added to each sample before the glucose measurements in order to comply with the linear range of the utilized biosensor. All measurements were conducted in triplicates.

\section{Results and Discussion}

\section{Design of a platform for sensor integration}

The platform presented was developed to address the current need of in/online monitoring of several components (e.g. product formation, cell viability and media composition) in bioprocesses. It was designed to be a part of a multi-component microfluidic platform, where each system is connected to others via a "plug and play" approach. Even though custom-made connections can be developed [22], the approach chosen in this work was aimed at developing a platform compatible with connection with external analytical devices and integration in the "plug and play" platform. Therefore, commercial finger tight fittings (such as, HPLC-type connectors) and standard tubing were used. The platform was built in order to be suitable for a wide range of applications. To this end, the final design, a closed channel, connected to a chamber where the sensor can be placed, enabled the required flexibility in terms of the sensor used and its easy replacement. The sensor is introduced in the platform through rectangular pockets which allow a wide variety of sensor shapes and widths to be integrated without requiring the modification of the whole platform. Furthermore, it is important to note that the sensor and platform fabrication are independent from each other. In this way, any sensor technology compatible with the platform material can be used, which considerably broadens the future opportunities for the platform's use.

The assembly of the microfluidic platform, performed using lamination with double-sided pressure sensitive adhesive tape (DAT), allowed a fast assembly of the different plates that 
form the platform (less than 15 minutes required), while also achieving a leakage-free flow and enabling the re-usability of the platform.

As proof-of-concept, screen-printed electrochemical sensors were chosen due to their cheap fabrication and wide use in microfluidic and health related applications. The possibility of applying these sensors as single-use detection elements is also an appealing concept due to the impending challenges of biofouling that bio-applications can generate during long-term measurements. Furthermore, two different types of electrodes were selected with the same detection principle and target, but with different materials and thicknesses. The choice of electrochemical sensors influenced the final design of the platform, presented in Figure 1 (b) and (c), due to the required sensing chamber shape. The distance between the measurement chambers (37.2 mm center to center) was defined based on the gap between the inlets of the interface connecting the sensors to the potentiostat used for measurement. In principle, due to the quick fabrication and assembly cycle of the platform $(<2 \mathrm{~h})$, adaptation to most types of sensors (by adjusting the sensing chamber) can easily be performed. The two integrated sensors are subjected to different flow rates during measurements, since one is positioned before the mixing/dilution chamber (first sensing chamber), while the other is positioned after the mixing/dilution unit (second sensing chamber). Thus, the first sensing chamber is only affected by the flow of one of the inlets (sample inlet), while the second sensing chamber, on the other hand, experiences a higher flow rate which is the sum of the flow rate from the two inlets (sample plus buffer for dilution). The overall internal volume of both designs is approximately $500 \mu \mathrm{l}$.

\section{Mixing and dilution unit}

In biological and biotechnological applications, variable substrate (or other components) concentration can occur, often within a wide range. A mixing/dilution chamber was thus added to the developed platform, allowing proper sample dilution for quantification to occur in a secondary sensor, when target samples are above the first sensor's concentration range. By diluting the matrix that reaches the detection chamber, the range of detectable concentrations of the integrated sensor is also broadened. The target sample concentration is diluted to the linear range measurable by the sensors, which is 0 to $5 \mathrm{mM}$ for graphene oxide-based sensors and 0 to $2 \mathrm{mM}$ of glucose for graphite-based sensors. The dilution platform, based on passive mixing, was designed and optimized following a similar concept to the one presented in Jeon and Shin [33]. Passive mixing was chosen to reduce dependence on external equipment. Several mixing designs were first simulated using a CFD software (ANSYS-CFX 16.2), before the best design was integrated in the platform and validated. The first designs were based on chaotic mixing generated by obstacles in the channel, since this approach provides fast mixing due to an increased interfacial contact between the different mixing phases [33], [34]. At microscale the flow is laminar and therefore predictable, simply requiring the solution of the Navier-Stokes equation without the use of turbulent models. The design of obstacles [35] within a chamber was preferred to a zigzag configuration (as presented in[33]) to avoid a high pressure drop [36] due to the sharp corners and extended length, which is relevant when developing a system for use in a series of chips. Also, to reach a design less prone to clogging, more complex mixing structures, such as Tesla structures, were avoided [37]. It is also 
important to consider when operating a passive mixer, that the mixing capability is highly dependent on the flow velocity. Therefore, the design must be optimized to achieve appropriate mixing at high flow rates. In Figure 2 all designs that include obstacles ((a) to (d)) are presented. It can be observed that the straight diagonal barriers design (Figure 2 (a)) had the best performance, especially at higher flow rates. However, even the structure showing the best performance was not capable of achieving the desired 1:9 dilution. Finally, a 3D serpentine channel was designed and simulated for the same flow conditions as shown in Figure 2 (e), resulting in the best mixing design. The pressure levels inside the final operating platform were lower than indicated by the pressure distribution obtained from the CFD simulations, since no security valve leakage at the pumps or leakage inside the platform was observed during the operation of the platform.

\section{Sensor characterization}

The main working principle of the biosensors applied in this study is electrochemical. It is based on the detection of either the substrate or the product of the enzymatic reaction, by measuring the changes in faradaic current that its oxidation or reduction causes on the surface of the working (sensing) electrode [38,39]. The magnitude of the measured current, obtained by applying a constant potential between the working and the reference electrodes, is usually proportional to the bulk concentration of the electroactive species present, hence enabling a quantitative output.

The sensors integrated in the developed microfluidic platform were characterized both in a static (droplet characterization) and in a dynamic environment (in-flow characterization). Both types of measurement were performed for the same substrate concentrations; and, in the case of the dynamic measurements, the flow rates used were identical to those that the sensors would be subjected to during the mixing/dilution experiment $(0.25$ to $10 \mu \mathrm{L} / \mathrm{s})$.

Figure 3 (a) and (b) present the measured calibration curves from the droplet chronoamperometry measurements. It can be observed that the graphite-based sensors (Figure 3 (a)) show a decreased linear range (until $1.7 \mathrm{mM}$ of glucose) with a slightly higher apparent sensitivity than the graphene oxide-based sensors (Figure 3 (b)). The latter sensors display a wider range of detection (up to $5 \mathrm{mM}$ of glucose) with a sensitivity less than 0.2 $\mu \mathrm{A} / \mathrm{mM}$. This difference in the sensor output can potentially be explained by the more uniform electrode surface provided by the graphene oxide-based SPEs together with the presence of an extra graphene oxide layer. Both sensor types were tested inside the platform and their dynamic response compared with the static approach.

In Figure 3 (c) and (d) the measured calibration curves for the dynamic method using the microfluidic platform are presented at different flow rates. This characterization was performed by introducing the same flow rate at the two inlets, and as a result the first sensing chamber was subjected to half the flow rate of the second sensing chamber. As illustrated in the figure, the graphite-based sensors (Figure 3 (c)) present a reduced linear behaviour than previously obtained in the droplet chronoamperometry, while the graphene oxide-based sensors (Figure $3(\mathrm{~d})$ ) show a decrease in the linear range (up to $\sim 4 \mathrm{mM}$ of glucose). Both 
sensor types however display a variation of the calibration curve with flow. A decrease of the sensitivity (slope), as well as of the detection range, is observed when the applied flowrate increases. The higher difference in static vs. flow behaviour observed for the graphite-based sensors can be related to the difference in electrode substrate (graphite instead of graphene oxide) as well as SPE substrate (thickness of polymeric layer) that can lead to a lower stability of the final graphite-based sensors. Furthermore, the higher surface area of the graphene oxide sensors enables the immobilization of higher amounts of both mediator and glucose oxidase, possibly contributing to the different output observed.

\section{Influence of flow}

The distribution of the current measured relative to the flow rates applied for the integrated graphite and graphene oxide-based sensors was also assessed. A similar trend can be observed for both, being however more pronounced in the graphene oxide-based sensors (Figure 4 (b)). At low substrate concentrations, a slight decrease of the measured current is observed with increasing flow rates. For concentrations higher than $1 \mathrm{mM}$, the opposite trend is observed, where the measured current increases with increasing flow rates. Both trends are however less prominent for flow rates higher than $5 \mu \mathrm{L} / \mathrm{s}$. The data corresponding to these observations is presented in Figure 3S in the Supplementary Information. Since the used flow rate and the substrate concentration affect the measured current, a study on the influence of flow on the sensor performance is required and data can only be analysed by using the calibration curves for each of the applied flow rates. Nonetheless, since the influence for the same type of sensor should be similar every time, such a calibration curve can be easily implemented in a monitoring software and allow for immediate data interpretation. Furthermore, since this behaviour is probably caused by the effect of flow on the diffusion layer above the sensor, it can also indicate which are the best flow conditions for the operation of the platform, or the flow conditions that are limiting.

\section{Continuous monitoring of diluted glucose solutions}

A successful integration of these sensors and the continuous monitoring of glucose samples in a microfluidic platform were achieved. Figure 4 presents the results of the mixing/dilution chamber test where the integrated sensors were used for continuous monitoring of diluted glucose solutions. Substrate quantification results of the outlet solutions obtained from HPLC measurements are also presented in Figure 5. The current measured at the sensors was converted into glucose concentration through the in-flow calibration curve (Figure 3 (c) and (d)) corresponding to the flow rate applied to the sensor (Table 1). As presented, the graphite and graphene oxide-based sensors have different sensitivities for the substrate, visible by their different responses, corresponding to their differences in sensitivity to glucose. However, both display a deviation from the HPLC data. The graphite-based sensor over-estimates the glucose concentration and the graphene oxide-based sensor under-estimates it, which can be caused by the influence of the flow rates on the glucose diffusion to the electrode surface, as explained previously. More specifically, the influence of the flow rate on the thickness of the diffusion layer above the sensor is expected to be the main limitation. Potential improvements to this deviation can be investigated with the aid of mathematical models and the previously mentioned fluid dynamics simulation. Such models allow accounting for existing concentration 
gradients formed inside the sensing chamber, diffusion limitations due to flow conditions, and the estimation of calibration curves for other flow rates, among others. Furthermore, the platform was capable of operating continuously for approximately $12 \mathrm{~h}$ in a wide range of flow rates, and with different sensor substrates and geometries. For each sensor type, the in-flow sensor calibration and dilution experiments in the assembled platform were performed sequentially and on the same day.

Regarding the mixing/dilution unit, the measured glucose concentrations at the outlet were higher than the expected concentration for the dilution used, with a variation of around 0.2 $\mathrm{mM}$. This margin of error is acceptable in biotechnological applications [40], [41].

\section{Evaluation of sensor performance with fermentation samples}

The performance of graphite glucose sensors with fermentation samples was also evaluated in preparation for integrating the glucose sensors on the developed microfluidic platform as an online monitoring tool in lab-scale fermenters.

Figure 5 (a) presents a comparison of the sensor calibration curve performed with pure glucose solutions (concentrations from 0 to $0.54 \mathrm{~g} \mathrm{~L}^{-1}$ multiplied by 50 in order to be in the range of glucose concentration in real fermentation samples) to a two-point calibration made with YPD medium spiked with $20 \mathrm{~g} / \mathrm{L}$ of glucose without microbial cells present (1:50 dilution of this solution was used to obtain the two-point calibration). As observed, the medium seems to affect sensor behaviour (sensor sensitivity is changed for the two-point calibration relative to the pure sample calibration), but not significantly. A small increase in the baseline current (point at $0 \mathrm{~g} / \mathrm{L}$ ) is also observed, and is expected considering the higher complexity and presence of a wider variety of compounds (e.g. other sugars) in the YPD medium relative to glucose solutions in PBS buffer. The performance of the sensor performance does not seem to be significantly affected by a complex fermentation medium.

Figure 5 (b) presents an at-line monitoring of glucose concentration during S. cerevisiae growth for $5 \mathrm{~h}$. It is possible to observe a slow decrease in substrate concentration in the first $3 \mathrm{~h}$ of growth followed by a significant decrease in the last $1.5 \mathrm{~h}$ monitored. Since all the presented points were acquired at a 1:50 dilution of the real sample, it was not possible to monitor the growth further. However, due to the low detection limit of these sensors, by gradually decreasing the sample dilution used, a very accurate determination of the point in time of glucose depletion was made. Even though the dilution applied was higher than the current sample dilution achievable with our platform, the monitoring performed of glucose concentrations in the $1 \mathrm{~mL}$ microbioreactor proved that the glucose biosensor developed can be used to for the determination of glucose in real samples.

\section{Conclusions and future work}

In this work, a microfluidic platform for continuous monitoring of substrate samples in biotechnological applications was developed. This platform involved the successful integration of two geometrically different electrochemical sensors and their application in continuous monitoring. The comparison between sensor output during static and dynamic measurements 
allowed the observation of a decrease in detection range for both sensors during in-flow measurements, relative to the droplet characterization. This change in the sensor's sensitivity derives from the fluid dynamic influence on the diffusion of target analyte to the electrode surface. Moreover, a significant difference in current output was also obtained at low and high flowrates, indicating that diffusion effects are highly relevant at this scale for the geometries employed. Finally, the platform displays the capability of performing sample dilutions of up to 10 times, even at high flow rates, through the use of an integrated mixing unit. This unit allowed tuning the sample concentration according to the sensor's detection limit, and can be improved further by increasing the number of bends in the meandering channel or by introducing some obstacles within the channel, which is large enough to implement such structures. Furthermore, fluid dynamic simulation tools (CFD) are currently being applied to the platform, focusing on the influence of the diffusion effects observed and how to account for them during platform data acquisition. An optimized chamber geometry, a flow rate range or operation and/or a mathematical relation between static and in-flow calibration curves are the expected outcomes of such fluidic simulations.

Graphite sensor performance with a complex medium was also evaluated, showing a small effect of the medium in both baseline current and sensitivity of the measurements. The sensors were also used to follow $S$. cerevisiae growth in a $1 \mathrm{~mL}$ bioreactor for $5 \mathrm{~h}$ by monitoring the glucose concentration at-line. The integrated sensors appear to be capable of handling real fermentation samples (after appropriate dilution and cell separation) and thus be applicable to on-line or in-line glucose quantification in lab-scale fermenters.

This platform may be further applied to different sensing technologies (e.g. optical, magnetic), due to the presented flexibility in terms of sensor format, as well as ease of assembly and possibility for re-use of both platform and integrated sensors. In addition, a combination of electrochemical and other sensor technologies (especially optical sensing) could be achieved on this platform, by using the predefined pockets for sensor integration. Likewise, the integrated mixing chamber can display a high variety of functions besides the demonstrated dilution ability. It may be further used as a reaction chamber for the screening of biocatalysts, as an inactivation chamber or as a labeling unit, by mixing the sample stream with a fluorescent or magnetic marker, depending on the sensing approach chosen.

The developed platform presented in this work exhibits a multi-function capability using a simple design, standard connectors and low-cost materials. Such a platform can contribute to increasing the number of bioprocess parameters measured online during monitoring, by enabling the integration of different sensor approaches, as well as their replacement, without the need to change the platform itself. The overall cost of such a platform makes it accessible both to research laboratories and industry. Additionally, the simple design and assembly method allows for mass production, by changing the fabrication method to e.g. injection moulding, and consequently being feasible for parallelization on a larger scale.

\section{Acknowledgments}


The authors thank the funding from the People Programme (Marie Curie Actions, Multi-ITN) of the European Union's Seventh Framework Programme for research, technological development and demonstration under grant agreement no 608104 (EuroMBR). The authors would like also to thank Tiago Pinto, Alexandr Zubov, Carina Gargalo and Tannaz Tajsoleiman for their inputs when writing this paper.

\section{References}

[1] Rameez S, Mostafa SS, Miller C, Shukla AA. High-Throughput Miniaturized Bioreactors for Cell Culture Process Development: Reproducibility, Scalability, and Control. Am Inst Chem Eng 2014;30:718-27. doi:10.1002/btpr.1874.

[2] Rohe P, Venkanna D, Kleine B, Freudl R, Oldiges M. An automated workflow for enhancing microbial bioprocess optimization on a novel microbioreactor platform. Microb Cell Fact 2012;11. doi:10.1186/1475-2859-11-144.

[3] Long Q, Liua X, Yanga Y, Lia L, Harvey L, McNeilb B, et al. The development and application of high throughput cultivation technology in bioprocess development. $\mathrm{J}$ Biotechnol 2014. doi:10.1016/j.jbiotec.2014.03.028.

[4] Mark D, Haeberle S, Roth G, von Stetten F, Zengerle R. Microfluidic lab-on-a-chip platforms: requirements, characteristics and applications. Chem Soc Rev 2010;39:115382. doi:10.1039/b820557b.

[5] Schäpper D, Alam MNHZ, Szita N, Lantz AE, Gernaey K V. Application of microbioreactors in fermentation process development: a review. Anal Bioanal Chem 2009;395:679-95. doi:10.1007/s00216-009-2955-x.

[6] Wu J, Gu M. Microfluidic sensing: state of the art fabrication and detection techniques. J Biomed Opt 2011;16:80901. doi:10.1117/1.3607430.

[7] Thrush E, Levi O, Cook LJ, Deich J, Kurtz A, Smith SJ, et al. Monolithically integrated semiconductor fluorescence sensor for microfluidic applications. Sensors Actuators, B Chem 2005;105:393-9. doi:10.1016/j.snb.2004.06.028.

[8] Billat S, Kliche K, Gronmaier R, Nommensen P, Auber J, Hedrich F, et al. Monolithic integration of micro-channel on disposable flow sensors for medical applications. TRANSDUCERS EUROSENSORS '07 - 4th Int Conf Solid-State Sensors, Actuators Microsystems 2007;146:29-32. doi:10.1109/SENSOR.2007.4300064.

[9] Nirschl M, Reuter F, Vörös J. Review of Transducer Principles for Label-Free Biomolecular Interaction Analysis. Biosensors 2011;1:70-92. doi:10.3390/bios1030070.

[10] Gärtner C, Kirsch S, Anton B, Becker H, Gmbh C, Promenade C, et al. Hybrid microfluidic systems - combining a polymer microfluidic toolbox with biosensors. Microfluid BioMEMS, Med Microsystems V 2007:2-5. doi:10.1117/12.715041.

[11] Becker H, Gärtner C. Polymer microfabrication technologies for microfluidic systems. Anal Bioanal Chem 2008;390:89-111. doi:10.1007/s00216-007-1692-2.

[12] Ehgartner J, Sulzer P, Burger T, Kasjanow A, Bouwes D, Krühne U, et al. Online analysis of oxygen inside silicon-glass microreactors with integrated optical sensors. Sensors Actuators B Chem 2016;228:748-57. doi:10.1016/j.snb.2016.01.050.

[13] Brusatori MA, Tie Y, Van Tassel PR. Protein Adsorption Kinetics under an Applied Electric Field: An Optical Waveguide Lightmode Spectroscopy Study. Langmuir 2003;19:5089-97. doi:10.1021/la0269558.

[14] Ricciardi C, Canavese G, Castagna R, Ferrante I, Ricci A, Marasso SL, et al. Integration of microfluidic and cantilever technology for biosensing application in liquid environment. Biosens Bioelectron 2010;26:1565-70. doi:10.1016/j.bios.2010.07.114.

[15] Park JW, Vahidi B, Taylor AM, Rhee SW, Jeon NL. Microfluidic culture platform for neuroscience research. Nat Protoc 2006;1:2128-36. doi:10.1038/nprot.2006.316. 
[16] Schaff UY, Xing MMQ, Lin KK, Pan N, Jeon NL, Simon SI. Vascular mimetics based on microfluidics for imaging the leukocyte-endothelial inflammatory response. Lab Chip 2007;7:448. doi:10.1039/b617915k.

[17] Wilhelm E, Neumann C, Duttenhofer T, Pires L, Rapp BE. Connecting microfluidic chips using a chemically inert, reversible, multichannel chip-to-world-interface. Lab Chip 2013;13:4343-51. doi:10.1039/c3lc50861g.

[18] Tkachenko E, Gutierrez E, Ginsberg MH, Groisman A. An easy to assemble microfluidic perfusion device with a magnetic clamp. Lab Chip 2009;9:1085-95. doi:10.1039/b812184b.

[19] Rapp BE, Schickling B, Prokop J, Piotter V, Rapp M, Länge K. Design and integration of a generic disposable array-compatible sensor housing into an integrated disposable indirect microfluidic flow injection analysis system. Biomed Microdevices 2011;13:90922. doi:10.1007/s10544-011-9560-4.

[20] Yang J, Yu JH, Rudi Strickler J, Chang WJ, Gunasekaran S. Nickel nanoparticlechitosan-reduced graphene oxide-modified screen-printed electrodes for enzyme-free glucose sensing in portable microfluidic devices. Biosens Bioelectron 2013;47:530-8. doi:10.1016/j.bios.2013.03.051.

[21] Gärtner C, Becker H, Carstens C, von Germar F, Drese KS, Fragoso A, et al. SmartHEALTH: a microfluidic multisensor platform for POC cancer diagnostics. In: Cullum BM, Porterfield DM, editors. vol. 7313, 2009, p. 73130B. doi:10.1117/12.820311.

[22] Hlawatsch N, Klemm R, Carstens C, Brandstëtter T, Becker H, Elbracht R, et al. A labon-a-chip system for the development of complex assays using modular microfluidic components. Microfluid BioMEMS, Med Microsystems X 2012;8251. doi:10.1117/12.910269.

[23] Gonçalves AM, Pedro AQ, Santos FM, Martins LM, Maia CJ, Queiroz JA, et al. Trends in protein-based biosensor assemblies for drug screening and pharmaceutical kinetic studies. Molecules 2014;19:12461-85. doi:10.3390/molecules190812461.

[24] Alonso-Lomillo MA, Domínguez-Renedo O, Arcos-Martínez MJ. Screen-printed biosensors in microbiology; A review. Talanta 2010;82:1629-36. doi:10.1016/j.talanta.2010.08.033.

[25] Mello LD, Kubota LT. Review of the use of biosensors as analytical tools in the food and drink industries. Food Chem 2002;77:237-56. doi:10.1016/S0308-8146(02)00104-8.

[26] Turner APF, Karube I, Wilson GS. Biosensors Fundamentals and Applications. vol. 53. New York: Oxford University Press, Walton Street, Oxford OX2 6DP Oxford; 1989. doi:10.1017/CBO9781107415324.004.

[27] Borgmann S, Schulte A, Neugebauer S, Schuhmann W. Amperometric biosensors. In: Alkire RC, Kolb DM, Lipkowski J, editors. Adv. Electrochem. Sci. Eng. Bioelectrochemistry, vol. 13, Weinheim: Wiley-VCH Verlag GmbH \& Co. KGaA; 2012, p. 50-4. doi:10.1016/S0958-1669(96)80094-2.

[28] Wang J. Electrochemical glucose biosensors. Electrochem Sensors, Biosens Their Biomed Appl 2008:57-69. doi:10.1016/B978-012373738-0.50005-2.

[29] Lyons MEG, Keeley GP. Carbon nanotube based modified electrode biosensors. Part 1.Electrochemical studies of the flavin group redox kinetics at SWCNT/glucose oxidase composite modified electrodes. Int J Electrochem Sci 2008;3:819-53.

[30] Karyakin AA. Prussian blue and its analogues: Electrochemistry and analytical applications. Electroanalysis 2001;13:813-9. doi:10.1002/15214109(200106)13:10<813::AID-ELAN813>3.0.CO;2-Z.

[31] Karyakin AA, Karyakina EE, Gorton L. On the mechanism of $\mathrm{H} 2 \mathrm{O} 2$ reduction at Prussian Blue modified electrodes. Electrochem Commun 1999;1:78-82. doi:http://dx.doi.org/10.1016/S1388-2481(99)00010-7. 
[32] Ricci F, Amine A, Palleschi G, Moscone D. Prussian Blue based screen printed biosensors with improved characteristics of long-term lifetime and $\mathrm{pH}$ stability. Biosens Bioelectron 2002;18:165-74. doi:10.1016/S0956-5663(02)00169-0.

[33] Jeon W, Shin CB. Design and simulation of passive mixing in microfluidic systems with geometric variations. Chem Eng J 2009;152:575-82. doi:10.1016/j.cej.2009.05.035.

[34] Lee C-Y, Chang C-L, Wang Y-N, Fu L-M. Microfluidic Mixing: A Review. Int J Mol Sci 2011;12:3263-87. doi:10.3390/ijms12053263.

[35] Suh YK, Kang S. A review on mixing in microfluidics. Micromachines 2010;1:82-111. doi:10.3390/mi1030082.

[36] Bhagat AAS, Peterson ETK, Papautsky I. A passive planar micromixer with obstructions for mixing at low Reynolds numbers. J Micromechanics Microengineering 2007;17:1017-24. doi:10.1088/0960-1317/17/5/023.

[37] Hong C-C, Choi J-W, Ahn CH. A novel in-plane passive microfluidic mixer with modified Tesla structures. Lab Chip 2004;4:109-13. doi:10.1039/B305892A.

[38] Chaubey A, Malhotra BD. Mediated biosensors. Biosens Bioelectron 2002;17:441-56. doi:10.1016/S0956-5663(01)00313-X.

[39] Thévenot DR, Toth K, Durst RA, Wilson GS. Electrochemical Biosensors: Recommended Definitions and Classification. Anal Lett 2001;34:635-59. doi:10.1081/AL-100103209.

[40] Nandakumar MP, Sapre A, Lali A, Mattiasson B. Monitoring of low concentrations of glucose in fermentation broth. Appl Microbiol Biotechnol 1999;52:502-7. doi: $10.1007 / \mathrm{s} 002530051552$.

[41] Lidgren L, Lilja O, Krook M, Kriz D. Automatic fermentation control based on a realtime in situ SIRE(R) biosensor regulated glucose feed. Biosens Bioelectron 2006;21:2010-3. doi:10.1016/j.bios.2005.09.012.

\section{Figure Legends}

Figure 1 - Schematics of the developed platform, highlighting the different elements and the sensor positions (a) and SolidWorks 3D representation of the assembled microfluidic platform with one integrated IS-C SPE (b), and (c) DRP-PW-110DGPHOX SPE

Figure 2 - Comparison of mixing capability between different mixing chamber geometries using CFD simulation for $1: 1$ dilution at $10^{-3} \mathrm{~m} / \mathrm{s}$, and of a 10 times difference between inlet velocities for two geometries (straight diagonal barriers channel and the 3D serpentine channel). In the figure the different geometries tested for mixing are presented: (a) straight diagonal lines, (b) squared baffles, (c) staggered herringbone, (d) combination of herringbone and obstacles and (e) three-dimensional serpentine channel

Figure 3 - Droplet amperometric characterization of the biosensors before integration in the microfluidic platform, (a) graphite-based sensors and (b) graphene oxide-based sensors, and in-flow amperometric characterization of the sensors used to test the microfluidic platform, (c) graphite-based sensors and (d) graphene-based sensors*

Footnote:* The graphite-based sensor used in the first chamber presented a high variability during in-flow characterization and so data is not presented 
Figure 4 - Comparison of diluted glucose concentration values measured online with the sensors and at the outlet using HPLC, for both types of SPEs used: (a) graphite-based sensors and (b) graphene oxide-based sensors

Figure 1 - Graphite sensor performance with fermentation samples: (a) pure sample calibration curve (dotted line with diamonds) vs. glucose spiked YPD media two-point calibration (dashed line with diamonds) and (b) at-line quantification of glucose consumption by $\mathrm{S}$. cerevisiae in a microbioreactor determined by a glucose biosensor $(\mathrm{N}=3)$.

Table 1 - Flowrates used to test the mixing/ dilution chamber inside the developed microfluidic platform.

\begin{tabular}{llll}
\hline Dilution & $\begin{array}{l}\text { Flowrate } \\
\text { second inlet } \\
\left(\mu \mathrm{L} \mathrm{s}^{-1}\right)\end{array}$ & $\begin{array}{l}\text { Flowrate in second } \\
\text { sensing chamber }\left(\mu \mathrm{L} \mathrm{s} \mathbf{s}^{-1}\right)\end{array}$ & $\begin{array}{l}\text { Flowrate used } \\
\text { for data } \\
\text { interpretation } \\
\left(\mu \mathrm{L} \mathrm{s}^{-1}\right)\end{array}$ \\
\hline $\mathbf{1 : 1}$ & 1 & 2 & 2 \\
$\mathbf{1 : 2}$ & 2 & 3 & 4 \\
$\mathbf{1 : 5}$ & 5 & 6 & 6 \\
$\mathbf{1 : 9}$ & 9 & 10 & 10 \\
\hline
\end{tabular}




\section{Supplementary Information:}

(a)

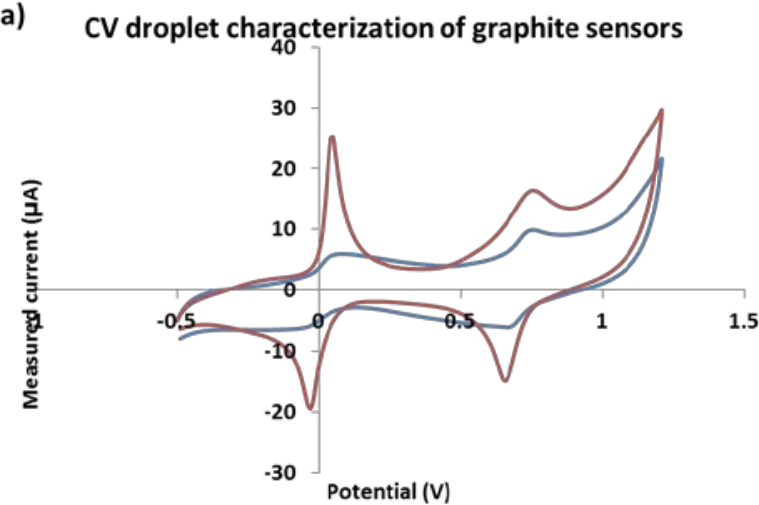

(b) CV droplet characterization of graphene oxide sensors

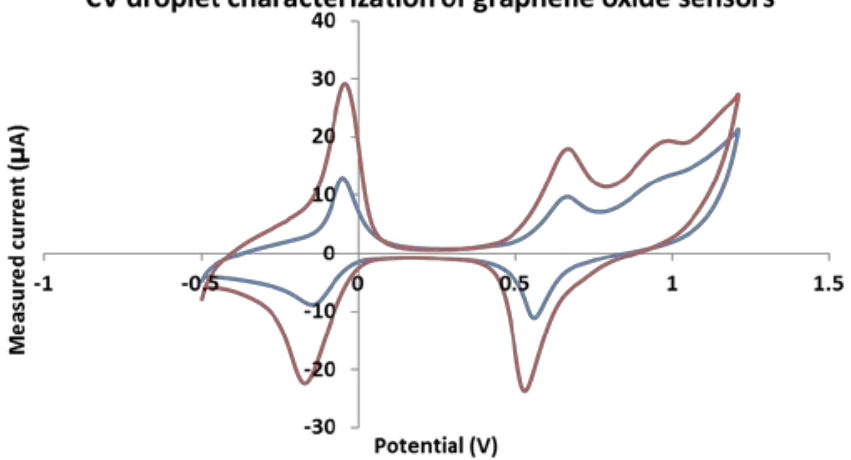

Figure 1S - Cyclic voltammograms of the sensors used for the microfluidic platforms test: (a) for the graphite-based sensors and (b) for the graphene oxide-based sensors. The sensor used in the first chamber is presented in blues, while the sensor used in the second chamber corresponds to the red curve.

(a)

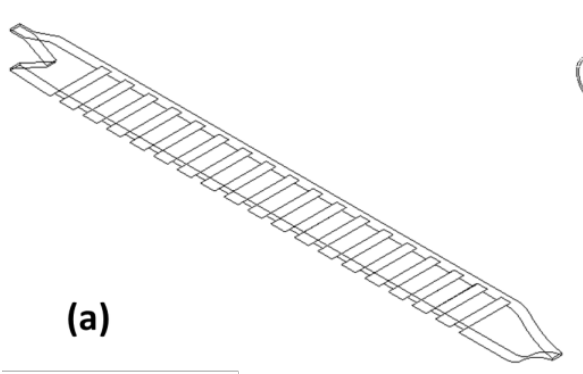

(b)
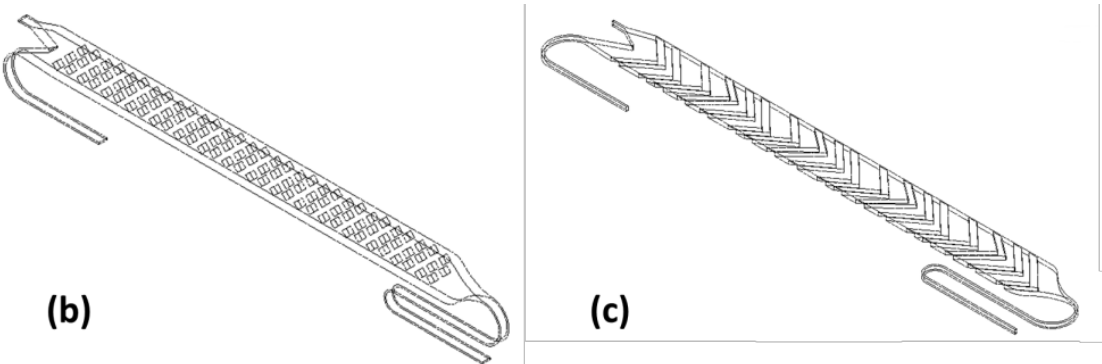

(d)

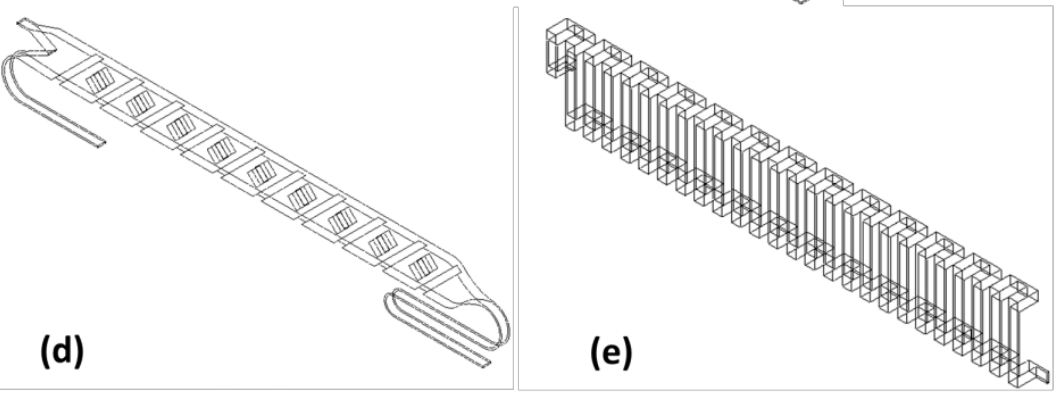

Figure 2S - Mixing chamber designs based on passive mixing structures: straight diagonal barriers (a), squared baffles (b), staggered herringbone (c) combination of herringbone and obstacles (d) and three-dimensional serpentine (e). 
(a)

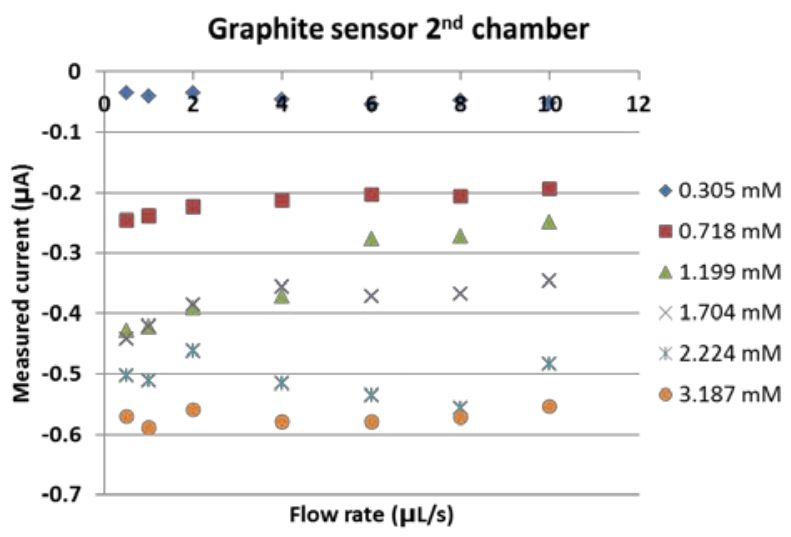

(b) Graphene oxide sensor $1^{\text {st }}$ Chamber

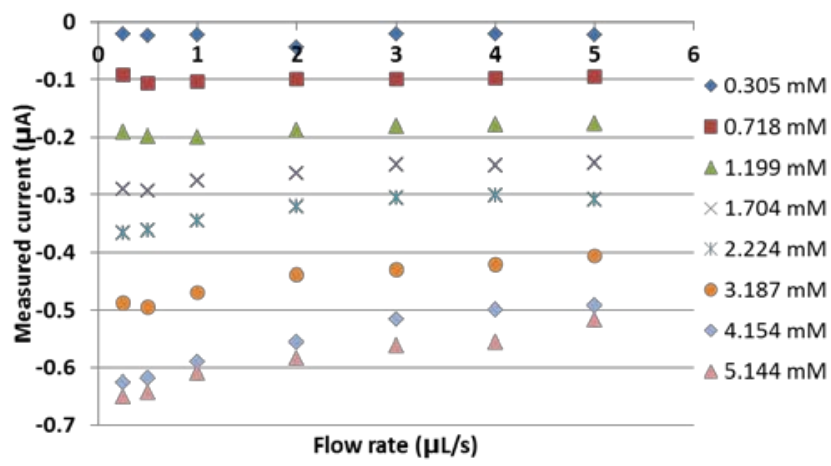

Graphene oxide sensor $2^{\text {nd }}$ Chamber

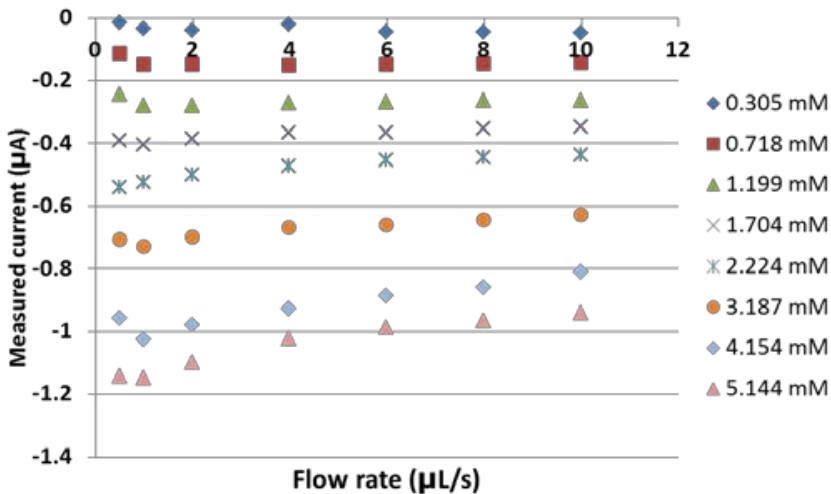

Figure 3S - Influence of flow on the current measured for the range of glucose concentrations tested for both types of sensors used: (a) graphite-based sensors and (b) graphene oxide-based sensors. 